\title{
Staying alive
}

More than $90 \%$ of the world population is infected with the Epstein-Barr virus (EBV), which predisposes carriers to various cancers by immortalizing host cells. A paper in Molecular Cell now identifies one mechanism that might explain the prolonged survival of EBV-infected cells.

Most DNA tumour viruses disrupt the function of the p53 tumour suppressor protein. But until recently, no EBV immortalizing proteins were known to act through p53. The EBV protein that is most likely to act on p53 is the widely expressed EpsteinBarr nuclear antigen (EBNA1), which has several important roles in latent EBV infection. EBNA1 binds stably to the ubiquitin-specific protease USP7, which was recently found to be associated with p53. Could EBNA1 affect the regulation of $\mathrm{p} 53$ by disrupting the p53/USP7 interaction? This hypothesis was further supported by the fact that USP7 binds and deubiquitinates p53 by using the same domain - the tumour-necrosis factor-receptor associated factor (TRAF)-like domain - that is bound by EBNA1. Now, Saridakis and colleagues provide convincing molecular evidence that this model is correct, thereby advancing a mechanistic explanation for the previously elusive tumorigenic properties of EBV.

The authors solved the crystal structure of the human USP7
$\mathrm{N}$-terminal domain both alone and when bound to an EBNA1 peptide. Using single anomalous dispersion, they found that the USP7 domain was structurally most similar to the TRAF-C domain of TRAF2. NMR chemical-shift mapping revealed that, from a molecular point of view, EBNA1 and p53 peptides bind to USP7 in a similar way, although the p53-USP7 complex had a smaller binding site and lower affinity than the EBNA1-USP7 complex. This provided definitive evidence that $\mathrm{p} 53$ and EBNA1 bind the same pocket on the USP7 molecule.

Further structural and biochemical studies showed that EBNA1 can disrupt $\mathrm{p} 53$ binding to USP7 by competing for the same site on USP7, so the team attempted to investigate the functional association between EBNA1 and p53. They transfected U2OS cells with plasmids expressing either EBNA1 or an EBNA1 mutant that cannot bind USP7 but is otherwise normal. These cells were then irradiated with ultraviolet to induce apoptosis. Fewer apoptotic cells were seen among the wild-type EBNA1 population compared with the EBNA1-mutant cells. Further transfection experiments confirmed that EBNA1 interfered with the stabilization of p53 by USP7.

The authors point out that the finding that EBNA1 indirectly destabilizes p53 is important for understanding the initial cell immortalization mechanism of EBV, the continual proliferation and survival of infected cells and their possible transformation to malignancy.

David Stevens

\section{(2) References and links}

ORIGINAL RESEARCH PAPER Saridakis, V. et al. Structure of the $p 53$ binding domain of HAUSP/ USP7 bound to Epstein-Barr nuclear antigen 1: implications for EBV-mediated immortilization. Mol. Cell 18, 25-36 (2005)

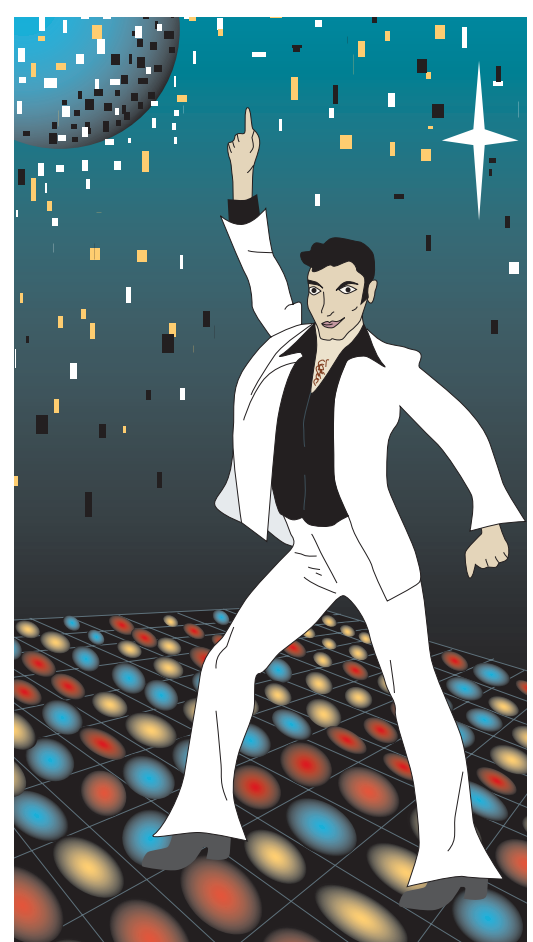

